\title{
57
}

\section{Developing Generic Capabilities through Web-based Student Profiling}

\author{
Wendy Fountain and Janelle Allison \\ Faculty of Built Environment \& Engineering, Queensland University of Technology, Brisbane, \\ Australia \\ w.fountain@qut.edu.au \\ School of Design \& Built Environment, Queensland University of Technology, Brisbaen, \\ Australia
}

Keywords: higher education, integration of ICT, World Wide Web, curriculum changes, assessment

\begin{abstract}
This paper reports on the development of the web-based Student Capability Profile (SCP) that is one institution's response to the increasingly complex professional, cultural, economic and social demands upon today's university graduates. This flexible and portable student profile facilitates and records students' generic capability or key skill development throughout the duration of study, anchored in core curriculum activity and drawing from other life experiences. Particular attention is given to the interdependence of the profiling process, its pedagogical framework, and the web and database technology that underpin the system. The implications and anticipated outcomes of the SCP's introduction are discussed and the SCP is finally considered as a catalyst for change impacting upon teaching practice and graduates' interface with society.
\end{abstract}

\section{INTRODUCTION}

With integration of information and communication technology (ICT), and namely the World Wide Web, frequently directed toward the delivery and support of courses and subjects in higher education, this paper describes an innovative application of web and database technology that transcends 
subjects, years and even entire courses. The emerging area of web-enabled student profiling for the purposes of facilitating and documenting the longterm development of generic capabilities or key skills is gaining momentum. The specific case of the Student Capability Profile (SCP) currently in development at Queensland University of Technology (QUT), Brisbane, Australia is a fusion of flexible learning and reflective practice principles, structured curriculum integration and the many opportunities afforded by the web medium's properties.

Profiling engages the student in a continuous monitored process of personal development facilitated by the web-based system and its human collaborators. Retained in possession of the individual, the capability profile is a documented attainment of capability synthesised from recordings and reflections of student experiences in all spheres of life, and tracked over the duration of study. As a process embodying the student's total experience, the SCP represents a major catalyst for change as graduates will begin to emerge from the institution with a more inherently connected range of disciplinary, technical and generic skills and knowledge.

The Student Capability Profile is a work-in-progress closely related to sister projects in three other Australian universities also in the membership of the Australian Technology Network (ATN). Planned for eventual implementation at institution level, two pilots are to extend from February to June, 2001 in the Faculty of Built Environment and Engineering. The background to the profile's development is presented below, followed by explanation of the relationship between the profiling process, its pedagogical framework and the properties of the web medium. The implications and anticipated outcomes of the profile's integration are then summarised.

\section{BACKGROUND}

An explanation of the perceived need for a student profile and its purpose reinforces the topical themes that are manifested within the concept of a student profile: graduate attributes, lifelong learning, integration of ICT to assist learning, and generic capabilities. Supporting students' personal development in the latter forms the central objective of the SCP. Generic capabilities may alternatively be termed 'key skills', 'generic skills' or 'transferable skills' in the literature, however distinctions are debated. These terms are all suggestive of the kinds of skill and knowledge identified by Ramsden (1992), the Dearing Report (1997) and Bowden and Marton (1998) among others, as extending beyond a discipline or subject area and which are applicable to all spheres of life. In the absence of a definitive list, such skills might include communication, interpersonal, and problem-solving skills; 
information, technological and critical literacies; and community, cultural and environmental consciousness.

The impetus for the development of student profiling in the Australian setting emerged from several quarters. Prominent among these was the AC Nielsen project (1999) that involved surveying employers to establish desired generic capabilities, and determining to what extent hired graduates displayed those capabilities. In short, the results revealed a sizable deficit in graduates' generic skills compared with their technical skills. Prior to this research, professional organisations such as the Institution of Engineers Australia (1996) became active in requiring universities to demonstrate how generic and technical skills were being cultivated. A further initiative that heavily informed the development of the SCP was the widely disseminated report prepared by the Australian Technology Network (ATN) entitled 'Generic Capabilities of ATN University Graduates' (1999). This report outlines a framework for generic capability development through curriculum integration and teaching practice, case studies of which are examined. Extending on the work of Bowden and Marton (1998), the report also raises critical distinctions in levels of generic capability attainment relative to their application in different contexts.

Examples of student profiling can also be found further afield. Of particular interest to the early stages of the QUT project, was the Universities of Liverpool, Manchester and Leeds' 'LUSID' project. Its aim was '...to implement a World Wide Web based system to reflect upon and record the outcomes of learning in a variety of contexts, including academic and non-academic activities'. The philosophy is further shared in the mission 'to reflect upon and record key skills development' in the undergraduate population (LUSID 2000). It contrasts most with SCP in its introduction as an optional tool outside of core curriculum activity, and its starting focus on capability rather than experiences that yield capability development. A further PC-based profiling approach is in use at Thames Valley University (1998) with emphasis on career planning in addition to key skill development.

\section{THE INTERDEPENDENCE OF PROCESS, PEDAGOGY AND MEDIUM}

The capability profiling process involves student interactions within a database-supported web environment, the pedagogical framework of which allows sufficient scope and flexibility for the teacher to customise and adapt system functions to achieve integration with core curriculum. This includes the creation of tasks, insertion of media and examples, linking of resources 
and exemplars, and the provision of access to appropriate mentors, guides and peers who also interact with students within the web environment.

In an orientation to the SCP students are prompted to undertake a 'selfaudit', rating themselves through reflection upon a range of life experiences. This self-assessment is made relative to the set of generic capabilities that are described and illustrated through exemplars in the system. Students are then assisted to identify areas in need of development, and opportunities for such development within core curriculum activities. Action then becomes explicit through the fundamental cyclical process of auditing, reflecting, and assessing. Students can also elect to release their profile to others within the system environment such as peers, mentors or tutors for the purposes of assessment, seeking feedback or collaborating.

It is envisaged that teachers would provide a greater degree of task structure for students early in the undergraduate time line, compared with third or fourth year students advanced in the creation of their capability profile. The notion that latter year students will utilise the profile more independently was supported in a recent focus group session with third year students. The important principle here is that regardless of year level, students can seek to demonstrate and record their attainment of a given generic capability at any point. The concern highlighted in the ATN Report (1999) is for the context in which the capability is to be demonstrated rather than any objectified 'satisfactory' level of capability. This factor recognises the diversity of background and prior learning students possess when embarking upon an academic program. Additionally, students are encouraged to support their profile with recognition of achievement in the community, the workplace or in study-related activities such as camps, school visits and special programs.

The resultant Student Capability Profile that augments a student's academic transcript upon graduation, is an extract of demonstrated generic capability attainment in the different contexts posed by course progression. In its attachment to the individual, the capability profile can flexibly accommodate changes of academic program, periods of overseas study, or be resumed after a deferment.

\section{PEDAGOGICAL PRINCIPLES AND IDEALS}

Several key pedagogical principles underpin the profiling process described above and have directed the way in which the web medium has been harnessed:

- Prompting individuals' consideration of their total engagement with the world, profiling promotes 'wholeness' i.e. a consciousness of the validity 
and value of all experiences and transfer of new skills to subsequent experiences

- Self-paced and personally constructed, students own both their learning process and the resultant capability profile, through which they are encouraged to develop a self-directed orientation to lifelong learning

- The system does not prescribe a path to a 'correct' end. Students are prompted to create unique paths based on their own experiences, and appreciate that the tutors, mentors and peers whom they encounter through the profile represent multiple perspectives

- While students interact with the system environment, their monitored actions are guided by human interventions that unfold as dialogue within the profile. This functions in the manner of Laurillard's (1993) rhetorical 'extrinsic feedback' upon which the student acts

- Recording and reflecting are central iterative processes that seek direct application in the context of curriculum objectives and activities, again conferring with Laurillard's learning schema (1993)

- Integration of the web-based profiling system is in itself conducive to the meta-cognitive development of a range of generic capabilities, especially in respect to technological and information literacies. Oliver and McLoughlin (1999) have raised this characteristic of web technology through their attempts to develop key skills over recent years. Supportive development of these highly transferable capabilities can contribute greatly to a student's ability to negotiate higher learning.

\section{PROPERTIES OF THE WEB MEDIUM}

Profiling in itself is not an innovation as the now dating texts describing paper-based approaches attest. What is exciting and compelling about the combined web and database medium is its conduciveness toward the pedagogical principles described above and to achieving a flexible framework. The major supportive properties include:

- Continuous access and functionality irrespective of time and location. Presuming web access, students and collaborators can log on 24 hours in principle, mindful that technical hitches will inevitably apply

- Database capabilities enable the recording, revisiting, organising and extraction of qualitative text input

- Levels of security allow students to activate the release of selected parts of their profile for task submission and additionally as desired, otherwise input remains personal and exploration need not be limited by scrutiny 
- In combination, secured access and the recording function allow authorised collaborators to generate an asynchronous text-based dialogue with students in support of a timely formative feedback turnaround

- References to shared events that occur outside the system in face-to-face scenarios, but are recorded inside the system by a tutor or peer, remain visible to reinforce the linkages between the curriculum activity and the related profile task

- The sophistication of current web authoring and graphics tools supports the creation of intuitive interfaces, a narrative graphic language and alternative prompt options that acknowledge diversity in learning and thinking styles, and an identifiable web environment with which students become familiar

- Ease of content exchange creates a responsiveness to changing emphases outside the system that demand particular skills e.g. a focus on flexible work practices within the new knowledge economy

- As a network node, the system makes explicit and meaningful connections between existing institution-based resources (such as online tutorials and guides that support information and technological literacy) that are otherwise passive and risk obscurity

- Viewing the network globally, the system acts as a gateway to a host of other web-based interactions and experiences that can be subject to meaning-making and testing upon return to the profile environment.

A further set of web medium properties converges with selected key 'learning paradigm' characteristics that MacFarlane (1997) regards as having superseded a passive, unidirectional and location-specific 'instructional paradigm':

- Interactivity is evoked by both system elements such as exemplars that may be activated, along with the input of human collaborators as action provokes response and feedback loops are generated

- In-built system support and examples of use are continuously available to avoid impeded progress reliant on human intervention

- Realistic bandwidth/file size solutions for dynamic media such as animation, audio and video are approaching and will result in more compelling exemplars, and concept mapping with minimised reliance on screen-based text.

Acceptance of the web medium's great opportunities as a platform in support of SCP cannot go unquestioned without examining some of its considerable challenges. Major implications are discussed below. 


\section{IMPLICATIONS OF SCP INTEGRATION}

As the web becomes an increasingly prevalent part of life it is easy to overlook that the Internet still poses some weighty equity issues for students. These include access for those with impairments, the inadequacy of oncampus computer facilities, the costs of online time and hardware for offcampus access, and deficits in the fundamental technical skills that allow participation. The integration of SCP with any student cohort demands a prestudy to first guarantee a realistic and equitable level of access and address alternatives if this cannot be achieved.

As suggested in discussion of the web medium's properties, there is potential for the SCP to become a more compelling experience through the integration of dynamic media. As web development in both the commercial sector and in education are beginning to address accessibility and usability, the conflict between providing high-end web applications and maintaining accessibility for the impaired user becomes very great. At a future point the institution will possibly have to consider the development of an alternative profile that seeks to meet all accessibility requirements while offering equivalent learning opportunities.

The objective to integrate the SCP institution-wide is an ambitious one, demanding something of a cultural revolution. Quite apart from huge resource and infrastructure implications, it asserts a new paradigm where the minimum standard of teaching includes rigorous integration of generic capabilities into core curriculum, and teaching with ICT, in addition to the previously accepted expertise in disciplinary knowledge and technical skills. This will clearly necessitate a lengthy process in which small scale integrations will gradually permeate outward. Such a shift will result as much from visionary leadership as policy, and specialised, dedicated educational development support. To complement this there will need to be equivalent infrastructure support for an eventually monstrous system with thousands of user accounts, and vast data management and archiving.

As the paradigm shift above suggests, there are many dimensions to the staff development that SCP will necessitate that extend far beyond learning to use and administrate the system. Negotiating the tension between what many see as achieving generic capability development at the cost of 'content' and vice versa, suggests that staff need assistance in reconceptualising these as being enmeshed in an interdependent whole. Once teaching with SCP, there will be the need to optimise one's approaches to moderating, mediating and providing meaningful feedback. Perhaps the most difficult concept with which the SCP developers and the institution still need to grapple is the substantive basis upon which students are verified as having attained levels of generic capabilities. 
In the expectation that employers, recruiters and professional bodies will understand, value and promote the SCP, they need to be regarded as part of a collaborative network and continue to inform the profile's iterative development. Workplaces will also form the sites of long-term evaluation when the first graduates who emerge with a SCP take up positions and research is conducted in how these graduates interface with professional workplaces.

Development and implementation of the SCP institution-wide is obviously a resource intensive endeavour. In the current climate of resource scarcity in higher education, commercialisation of the SCP has already been mooted to recoup costs and capitalise on the investment made in its development. The development of LUSID (2000) was coupled by a similar intention to make the system available to other institutions, and more lately the focus has turned to small to medium enterprises without personnel or staff development units. At QUT, alumni have been identified as a potential 'market' but all such pursuits under the model of economic rationalism require vigilance to avoid the day when the SCP becomes a product rather than a scholarly approach to teaching and learning.

\section{ANTICIPATED OUTCOMES}

In the first instance students will come to understand the limitations of discipline-only knowledge and technical skills, and appreciate the significance and value of generic capabilities. Students will also:

- Come to conceptualise the interdependence of all life experiences

- Consciously identify their own needs and make explicit the development they derive from curriculum and other activities

- Develop the skills to engage in effective peer mentoring of students in earlier stages of development

- Move from being supported to taking responsibility for their own longterm development

- Make connections between bodies of knowledge that were previously viewed in isolation, and understand the multidisciplinary nature of professional activity

- Participate in and adapt to changing economic and social conditions, and be equipped for multiple career paths and complex cross-cultural interactions.

Aside of the inherent challenges, positive outcomes are also anticipated for staff. It is an unrealistic expectation that all staff will represent a consistently high level across a diverse spectrum of generic capabilities. The SCP integration presents the opportunity for concurrent personal development 
and knowledge sharing through the formation of complementary 'skill teams'. The SCP will likely represent an initial foray into teaching with ICT for many staff. Favourably this will occur with formal support at institution level, and from an increasing collegial support network as the integration permeates. There will also be the opportunity to keep pace with advances in web and database technology as it is appropriated into the system.

\section{CONCLUSION}

Integration of the SCP at QUT is a catalyst for change at many levels. In the student experience it will result in improved outcomes for learning, personal growth and development; while in the staff experience it will also redefine teaching practice and priorities. It will also challenge and advance curriculum design and integration, and through this process advance the scholarship of teaching. Simultaneously the SCP will reveal and make common the experience of teaching with ICT in contrast to the relatively isolated pockets of ICT application that exist today. As an integration of ICT into higher education, the SCP is a multi-faceted catalyst for change in its relationship to students, staff and the institution.

\section{REFERENCES}

Nielsen AC (1999) Employer satisfaction with graduate skills: research report. [www.detya.gov.au/highered/eippubs/EIP99-7/execsum99_7.htm] 24.10.2000.

Bowden, J., Hart, G., King, B., Trigwell, K. and Watts, O. (1999) Generic capabilities of ATN university graduates. [www.clt.uts.edu.au/ATN.grad.cap.project.index.html] 24.10.2000.

Bowden, J. and Marton, F. (1998) The University of Learning: Beyond Quality and Competence. Kogan Page, London.

Dearing Report (1997) Higher Education in the Learning Society. HMSO, London.

Institution of Engineers Australia (1996) Changing the culture: Engineering education into the future. Institution of Engineers Australia, 21 Bedford Street, North Melbourne, Australia, 3051.

Laurillard, D. (1993) Rethinking University Teaching: A Framework for the Effective Use of Educational Technology. Routledge, London.

LUSID (2000) LUSID final report, May 2000-Lessons learned and future activity. [http://lusid.liv.ac.uk/public/report2000/lessons.html] 18.10.2000.

MacFarlane, A. G. W. (1997) New learning paradigm: Changes in pedagogy. In Towards a learning strategy for the University of the Highlands and Islands, L. Broumley, ed. [www.uhi.ac.uk/LET_Report.html\#chapter2] 17.10.2000.

Oliver, R. and McLoughlin, C. (1999) Using web and problem-based learning environments to support the development of key skills. In Proceedings of the Conference of the Australasian Society for Computers in Learning in Tertiary Education, J. Winn (ed.), Brisbane, December. pp.249-256. 
Ramsden, P. (1992) Learning to Teach in Higher Education, Routledge, London.

Thames Valley University (1998) Profiling User Guide, Thames Valley University, London.

\section{ACKNOWLEDGEMENTS}

Great thanks to Martin Murray, Doug Hargreaves, Jill Franz, Anne-Marie McArdle, Gail Hart, Halima Goss and SMILE, Weilin Chang and the QUT students of Civil Engineering and Interior Design.

\section{BIOGRAPHY}

Wendy Fountain is an educational designer and is currently coordinating the redevelopment of a faculty web environment. She has taught in the vocational, corporate and higher education sectors in Australia and Sweden, and has researched the experience of students and staff within ICT integration. Her current research interests are the qualitative aspects of online learning environments and web usability in respect to cognition and learning.

Janelle Allison is currently Interim Head of the new School of Design and Built Environment. Her research and teaching interests focus on regional planning and local economic development, and she has received both grants and consultancies on the role of local government in local economic development, community development, and industry clusters and networks. Her recent work has focussed on a new innovative learning communities approach to community development strategies. 